\title{
Review of: "High resolution species detection: accurate long read eDNA metabarcoding of North Sea fish using Oxford Nanopore sequencing"
}

\author{
Toshifumi Minamoto ${ }^{1}$
}

1 Kobe University

Potential competing interests: The author(s) declared that no potential competing interests exist.

The manuscript entitled "High resolution species detection: accurate long read eDNA metabarcoding of North Sea fish using Oxford Nanopore sequencing" by Doorenspleet et al. reported the field application of long read eDNA metabarcoding for fish species in the North Sea.

In eDNA metabarcoding, which has developed rapidly in recent years, relatively short gene fragments have been used as markers, but there is a room for improvement in terms of resolution. In this study, by using Oxford Nanopore, the authors succeeded in eDNA metabarcoding using markers with a length of 2,000 bp. In addition, the low sequencing accuracy, which is a weak point of the Oxford Nanopore, was successfully compensated by the newly developed pipeline Decona, and accurate long read sequencing was achieved. This research is of great value because it provides a new and powerful tool in the field of eDNA metabarcoding.

However, I think that this manuscript needs to be revised. The main problem is that the research methodology is not fully described. For example, Lines $126-128$ say that $2 \mathrm{~L}$ water samples were collected, but it does not say how many samples were collected. In addition, Figure 1a shows "Lab 4X" and "Field 1X", but I am not sure what the "Lab" and "Field" here refer to. Since it is difficult to follow what samples were obtained and how they were processed, it would be helpful to make a table that summarizes the samples and how they were processed. Also, there should be some way to identify the name of the software or pipeline used in this study. For example, italicize the name, or start with a capital letter. For example, the program name Medaka is sometimes written as "Medaka" or "medaka", which is confusing.

Another point of concern is the setting of the threshold for species identification. The authors set $99 \%$ as the threshold for identifying a species, but is this reasonable? Previous eDNA metabarcoding studies have used more relaxed criteria. Of course, the threshold does not have to be the same, since the new longer marker is likely to contain more conservative regions than previous shorter metabarcoding markers, but I would like to see verification of the validity of the identification threshold. 
